\title{
REGIONAL VARIATION IN FLUID RESERVOIR THICKNESS, OXYGEN TRANSMISSIBILITY AND CORNEAL OEDEMA DURING SCLERAL LENS WEAR Chukwuemeka Junior Obinwanne,OD, FAAO, FSLS ${ }^{1}$; Damian C. Echendu, OD, MSc ${ }^{1}$; Isaura llorena d'Alva Brito dos Santos, B.Optom, FAAO2; Sharon Onwuka, OD ${ }^{1}$; Osazee Agbonlahor, OD, MPH \\ ${ }^{1}$ Cornea and Contact Lens Unit, De Lens Ophthalmics Family and Vision Care Centre, Abuja, Nigeria ${ }^{2}$ Department of Optometry, University of Lurio, Nampula, Mozambique
}

Corresponding Author: Chukwuemeka Junior Obinwanne: obinwannejr@gmail.com

Submitted: 8 September 2021. Accepted: 24 November 2021. Published: 17 December 2021.

\begin{abstract}
Purpose: To determine if regional variation in post lens fluid reservoir thickness (PLFT) during scleral lens wear leads to regional variation in oxygen transmissibility and corneal edema during 4 hours of nonfenestrated scleral lens wear.

Methods: About 20 healthy subjects (mean age, $28.8 \pm 4.2$ years) were fitted with nonfenestrated rotationally symmetric scleral lenses. Anterior segment optical coherence tomography was used to measure cornea thickness before and after lens wear, PLFT 10 minutes and 4 hours after lens application, and scleral lens thickness (with the scleral lens in situ) 4 hours after scleral lens application. These measurements were limited to the central $6 \mathrm{~mm}$ and divided into three zones (central, mid- peripheral, and peripheral zones). In the mid-peripheral and peripheral zones, eight principal meridians were measured, generating 17 measurement points in total. Scleral lens thickness and PLFT measurements were corrected for optical dis-tortions by a series of equations. Oxygen transmissibility was calculated by dividing the scleral lens oxygen permeability by the optically-corrected scleral lens thickness, taking into account the oxygen permeability of saline and fluid reservoir thickness.

Results: A significant regional variation in PLFT $(F=12.860, P=0.012)$ was observed after 10 minutes of the lens application, PLFT was thickest and thinnest in the inferotemporal and the superonasal region of the peripheral zones( $322.6 \pm 161.8 \mu \mathrm{m}$ and $153.8 \pm 96.4 \mu \mathrm{m}$, respectively); however, this variation was not statistically significant at 4 hours of scleral lens wear $(\mathrm{F}=4.692 ; \mathrm{P}=0.073)$. Despite significant regional variation in oxygen transmissibility $(\mathrm{F}=48.472 ; \mathrm{P}=0.001)$ and relatively low oxygen transmissibility through the scleral lens, induced corneal edema did not vary significantly in different regions $(\mathrm{F}=3.346$; $\mathrm{P}=0.126)$. In the central corneal region, the induced corneal edema correlated moderately with PLFT $(\mathrm{r}=$
\end{abstract}

J Cont Lens Res Sci Vol 5(1):e32-e43; 17 December 2021

This article is distributed under the terms of the Creative Commons Attribution-

Non Commercial 4.0 International License. (C2021 Obinwanne CJ et al. 
$0.468 ; \mathrm{P}=0.037)$ and oxygen transmissibility $(\mathrm{r}=-0.528 ; \mathrm{P}=0.017)$. This relationship was insignificant in the peripheral cornea.

Conclusion: The inferotemporal peripheral region had the thickest PLFT and least oxygen transmissibility, and the superonasal region had the vice versa. Despite significant variation in PLFT and oxygen transmissibility initially, in healthy corneas, this variation does not seem to induce statistically significant regional variation in corneal edema. Increased central PLFT and decreased oxygen transmissibility moderately correlate with central corneal edema.

Keywords: corneal oedema; oxygen permeability; oxygen transmissibility; scleral lenses; tear reservoir thickness

\section{INTRODUCTION}

The fluid reservoir underneath the scleral lens corrects corneal irregularity in ectatic corneal diseases such as keratoconus, pellucid marginal corneal degeneration, and high corneal astigmatism. ${ }^{1}$ It also provides symptomatic relief and an ecosystem for severe dry eye management and ocular surface disease. ${ }^{1}$ Though scleral lenses were first described at the end of the 19th century, their clinical use was limited because of manufacturing limitations and poor oxygen permeability, which could cause corneal hypoxia. ${ }^{2}$

Gas permeable materials were first described in scleral lens manufacture in $1983^{3}$; however, the thickness of the modern scleral lens and the fluid reservoir underneath it still create a barrier to oxygen available to the cornea. ${ }^{4}$ These factors can cause corneal oedema ${ }^{5}$ because of anaerobic metabolism and a change in the ion concentration within the cornea in a hypoxic environment. ${ }^{6}$ The corneal endothelium maintains corneal hydration through the pump-leak mechanism. ${ }^{7}$ But a recent study demonstrated that after 25 minutes of scleral lens wear, the endothelium itself develops blebs, ${ }^{8}$ and scleral lenses fitted in post graft corneas has been shown to induce significant corneal edema. ${ }^{9,10}$

Studies also show that scleral lens-induced corneal edema is stromal and peaks at about 90 minutes after lens application; ${ }^{11}$ although interindividual differences in the magnitude of corneal edema induced by scleral lenses exist. ${ }^{12}$ Kim et al.'s ${ }^{13}$ study on central corneal swelling showed that scleral lens wear with post lens fluid reservoir thickness (PLFT) of less than 400 microns produced less than $2 \%$ central corneal swelling. Based on their oxygen transport modeling calculations, a change in PLFT from 50 microns to 400 microns increased corneal swelling by only $1-1.5 \%$, with oxygen transmissibility greater than $10 \mathrm{~h}$ Barrer/cm. Similarly, another study that evaluated the effect of varying oxygen permeability (Dk of ranging from 32 to 115) and scleral lens thickness (ranging from 0.15 to $1.20 \mathrm{~mm}$ ) on central corneal swelling reported that the increase in oxygen permeability and decreasing scleral lens thickness (thereby increasing oxygen transmissibility) showed a significant decrease in central corneal edema. ${ }^{5}$ However, this study found the relationship between cornea edema and oxygen transmissibility was nonlinear for high levels of oxygen transmissibility. Not surprisingly, several other studies showed that scleral lenses with high oxygen permeability fit with a fluid reservoir thickness of $\leq 300 \mu \mathrm{m}$ induced corneal edema of less than $2 \%$ after $3-8 \mathrm{~h}$ of scleral lens wear. ${ }^{14,15}$ This amount of corneal edema maybe considered minimal compared with corneal swelling induced by eye closure when asleep. ${ }^{14}$

Nevertheless, there is an undeniable relationship between the level of oxygen available to the cornea, the PLFT underneath the scleral lens, and the oxygen transmissibility of the lens. Theoretically, the oxygen tension available at the cornea surface decreases with higher PLFT in healthy eyes. ${ }^{16} \mathrm{Nau}$ 
et al. ${ }^{17}$ reported a significant decrease in PLFT after 2 hours of scleral lens wear with the PLFT in the inferior region within the central $2 \mathrm{~mm}$ of the area examined, showing the greatest decrease in PLFT (54\%), and the least was noted in the temporal region. Vincent et al. ${ }^{18}$ reported that tilting of the optic zone in the central $6 \mathrm{~mm}$ of the scleral lens, with the greatest asymmetry of PLFT, was noted in the horizontal meridian of the scleral lens (nasal vs. temporal regions) followed by the superior nasal vs. the inferotemporal region.

No clear understanding is still available as to how the regional variation in scleral lens PLFT leads to regional variation in oxygen transmissibility and corneal edema induced by scleral lens wear. Hence, this study aimed to determine the presence (or absence) of regional variation in fluid reservoir thickness and oxygen transmissibility during scleral lens wear and whether such variation was associated with regional corneal edema variation.

\section{METHODS}

This prospective study involved 20 eyes of 20 healthy participants (12 females and eight males; age, $28.8 \pm 4.2$ years). The study approval was obtained from the Health Research Ethics Committee of the Federal Capital Territory Administration (FCTA) Abuja, Nigeria, and followed the tenets of the Declaration of Helsinki. Participants recruited were residents of the Gudu district in Abuja, Nigeria and they provided written informed consent.

The study included participants with spherical equivalent refractive error of $\pm 4.00 \mathrm{D}$, no ophthalmic or systemic disease, intraocular pressures between 10 and $20 \mathrm{mmHg}$, no history of contact lens wear, or previous ocular surgery were included. One eye was randomly selected to be used for the study. The procedures were divided into two visits: the first visit was the eligibility visit and scleral lens trial; the second visit involved measurement of the finalized scleral regional PLFT at 10 minutes and 4 hours after wear and measurement of the scleral lens thickness.

\section{Scleral lens trial}

Scleral lens fitting was done by a single examiner using a nonfenestrated spherical $16 \mathrm{~mm}$ scleral lens (Europa, Visionary Optics Inc., Boston XO, USA) with a center thickness of $400 \mu \mathrm{m}$ and oxygen permeability (Dk) of 100 Fatt Dk units obtained from a diagnostic set. A 46D base curve lens, with a sagittal height of $4660 \mu \mathrm{m}$ was applied as the initial lens based on the manufacturer's guide for fitting regardless of keratometry readings; if the initial central PLFT exceeded 400 microns based on slit-lamp evaluation using the lens thickness as a reference, the sagittal height of the lens was decreased, and if the central PLFT was less than 100 microns, the sagittal height was increased. After 30 minutes of settling, the fit was reassessed and adjusted if required. Anterior segment optical coherence tomography (AS-OCT) (Optovue Inc, CA, USA) was used to measure the PLFT. A lens fit was deemed acceptable if it met the following criteria after 1 hour of setting:

1. Central and limbal PLTT was $\leq 350$ and $<150$ microns, respectively, based on AS-OCT assessment.

2. Absence of blanching or impingement of conjunctival vessels and no edge lifts.

3. Absence of corneal or conjunctival staining, no conjunctiva impression or epithelial edema on lens removal.

The lens placed in the study eye had a median power of of $-0.50 \mathrm{DS}$ (range, 0.00 to $-2.50 \mathrm{DS}$ ). The finalized lens was used in the second visit of this study. The corneal thickness was measured one day after the first visit after at least 3 hours after waking up and 4 hours of scleral lens removal using the AS-OCT device, respectively. This device showed eight radial line corneal scans, at a $6 \mathrm{~mm}$ length with 1024 A-scans per line over $6 \mathrm{~mm}$ corneal diameter divided into three zones with reference from the center: $0-2 \mathrm{~mm}$ (central zone); 2-5 mm (mid-peripheral zone); and 5-6 mm (peripheral zone) (Figure 1). The mid-peripheral and peripheral zones were evaluated in the eight corneal principal meridians: superior, superonasal, nasal, 
inferonasal, inferior, inferotemporal, temporal, and superotemporal, totaling 17 measurements of PLFT, scleral lens thickness, and corneal thickness for each AS-OCT scan. Hence the comparison of corneal edema, scleral lens thickness, and PLFT was performed in these zones. Participants fixated on a target within the device and a circular reticule was centered within the pupil visible through the preview monitor to ensure the same area was scanned each time.

Scleral lens thickness and PLFT were measured using the caliper tool in the AS-OCT device by using the minimum distance method. ${ }^{19}$ This method measures the minimum distance between 2 opposing layers and has been previously used in other studies in measuring ocular tissue thickness. ${ }^{20,21}$ The caliper tool method is less prone to errors than the popular axial method and yields measurements close to the LaPlace method (a more mathematically complex and reliable method). ${ }^{19}$

The PLFT was determined by measuring the shortest distance between the inner surface of the posterior scleral lens to the anterior corneal surface in the three zones and 17 regions. The PLFT measurements were performed in the following zones: (i) primarily in the central zone; (ii) two locations equidistant from the central PLFT in the mid-peripheral zone; and (iii) two locations, one on each
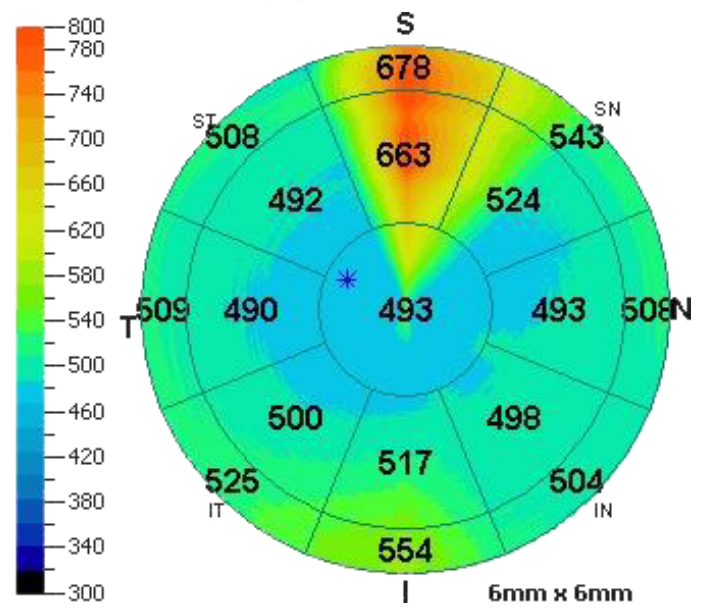

FIGURE 1. Anterior segment optical coherence tomography pachymetry map showing the 17 corneal regions over the central $6 \mathrm{~mm}$ cornea. side equidistant from the cornea central in the peripheral zone (Figure 2). This measurement was repeated for four meridians at 10 minutes and 4 hours intervals with a scleral lens in situ; thereby, generating 17 PLFT in the 17 regions. Scleral lens thickness was also measured similarly. On-eye scleral lens thickness measurement allows for the lens thickness measurement, PLFT, and change in corneal thickness in the same region.

The scleral lens thickness and PLTT measured with the AS-OCT device is an apparent image and can be affected by the lens curvature, the refractive index of the scleral lens material, and spatial distortions for noncentral locations. The equation series proposed by Ramasubramanian and Glasser ${ }^{22}$ was originally employed to correct optical distortions in soft contact lens thickness measured by the Visante AS-OCT was used to correct the raw scleral lens thickness and PLFT measured with the AS-OCT.

Equation (1) was used to determine the curvature of the front scleral lens power, Equation (2) to determine the vergence to the image of the posterior scleral lens surface after refraction by the front scleral lens surface, Equation (3) was used to determine the vergence of the posterior scleral lens surface before refraction by the front surface of the scleral lens, and lastly, the actual corrected scleral lens thickness was calculated using Equation (4) as the distance before refraction by the front surface of the scleral lens to the actual posterior scleral lens surface.

$$
F_{s c l}=\frac{1000\left(n_{\text {BostonXO }}-n_{\text {air }}\right)}{r_{f s}}
$$

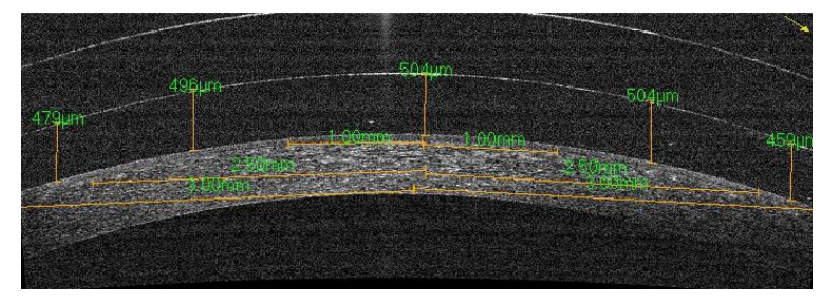

FIGURE 2. Measurement of PLTT in the different corneal regions and zones over the central $6 \mathrm{~mm}$ cornea. 
where $F_{s c l}$ is the power of the front scleral lens surface, $n_{\text {BostonXO }}$ is the refractive index of the scleral lens material, which is $1.415, n_{\text {air }}$ is the refractive index of air which is 1.00 , and $r_{\mathrm{fs}}$ is the radius of curvature of the front surface of the scleral lens.

$$
L^{\prime}=\frac{1000 \times\left(n_{\text {BostonXO }}\right)}{l^{\prime}}
$$

where $L^{\prime}$ is the dioptric image vergence and l' is the AS-OCT measured scleral lens thickness in millimeters (mm).

$$
\begin{gathered}
L=L^{\prime}-F_{s c l} \\
l=\frac{1000 \times n_{\text {air }}}{L}
\end{gathered}
$$

where $l$ is the optically corrected scleral lens thickness and $n_{\text {air }}$ is the refractive index of air which is 1.00 .

The same equation series was used to calculate the corrected PLFT. However, in Equation (1), instead of calculating the $F_{s c l}$ alone, the effective power $F_{\text {total }}$ was calculated (Equation [5]). This was determined by first calculating the front surface power of the PLFT $\left(F_{P L F T}\right)$ alone (which was negative because of movement from a high to lower refractive index surface, i.e., the scleral lens to tear reservoir), and then adding this to the front surface power of the scleral lens $\left(F_{s c l}\right)$ as it plays a role in the magnification of the PLTT front surface power; this final value was deemed the $F_{\text {total }}$. Secondly, refractive index of the tear reservoir system used was 1.334 (refractive index of saline) in place of the refractive index of air.

$$
\begin{gathered}
F_{P L T T}=\frac{1000\left(n_{\text {saline }}-n_{\text {BOSTON }}\right)}{t_{b s}} \\
F_{\text {total }}=F_{P L F T}+F_{\text {scl }} .
\end{gathered}
$$

where $F_{P L F T}$ is the power of the front surface of the PLTT and $F_{\text {total }}$ is obtained by adding the $\mathrm{F}_{\text {PLFT }}$ to the $\mathrm{F}_{\text {scl }}$. After obtaining the $F_{\text {total }}$, the equation is then followed sequentially from Equations 2-4, replacing $n_{\text {Boston }}$ with $n_{\text {saline }}$. The final l-value obtained in Equation (4) represents the corrected PLFT.

The oxygen transmissibility (Dk/t) of the scleral lens-tear reservoir system in different regions was calculated using the resistance formula previously proposed by Weismman et al., ${ }^{23}$ used to calculate the oxygen transmissibility of a piggyback contact lens system. Later in 2012, Langis et al. ${ }^{24}$ reused this equation in calculating transmissibility through a scleral lens (Equation 6).

$$
D k / t_{S C L}=\frac{1}{\left(t_{1} / D k_{1}\right)+\left(t_{2} / D k_{2}\right)}
$$

where $D k / t_{S C L}$ is the oxygen transmissibility of the scleral lens tear reservoir system, $t_{1}$ is the optically corrected thickness of the scleral lens (cm), $D k_{1}$ is the oxygen permeability of the scleral lens (100 Fatt Dk units), $t_{2}$ is the optically corrected PLFT (cm), and $D k_{2}$ is the oxygen permeability of saline (assumed to be 80 Fatt Dk units. ${ }^{25}$

\section{Statistical analysis}

Data normalcy was determined using ShapiroWilk's test. The difference in the corneal thickness before and after scleral wear in each corneal region was tested for statistical significance using the paired Student's t-test. The variations in the corrected PLTT at 10 minutes and at 4 hours of scleral lens wear and corneal edema at different regions, scleral lens thickness in the $6 \mathrm{~mm}$ region of the scleral lens, and the $D k / t$ of the scleral lens tear reservoir system were analyzed by analysis of variance. Post hoc pairwise comparison was conducted using the Bonferroni correction when indicated.

Pearson correlation analysis determined the relationship between regional PLTT, regional corneal thickness, tear reservoir system oxygen transmissibility, and corneal edema. A P value of $<0.05$ was considered statistically significant. Data are presented as mean \pm standard deviation (SD). SPSS Version 22.0 (IBM Corps, Armonk, NY) was used to carry out all statistical analyses. 


\section{RESULTS}

\section{Regional variations in post lens tear reservoir thickness}

A significant regional variation in PLFT was noted 10 minutes after scleral lens application $(\mathrm{F}=12.860 ; \mathrm{P}=0.012)$ in the central $6 \mathrm{~mm}$ of the scleral lens. On comparing the PLFT in the central 0-2 mm zone, the PLFT variation was statistically significant in 11 regions. However, this variation was not significant in five regions (the inferonasal and superotemporal region in the $2-5 \mathrm{~mm}$ zone; the inferior, inferotemporal, inferonasal, and the superotemporal regions of the 5-6 $\mathrm{mm}$ zones). The thickest PLFT was noted in the inferior temporal region of the 5-6 $\mathrm{mm}$ zone of the scleral lens (mean [SD], 322.6 [161.8] $\mu \mathrm{m}$ ), and the thinnest PLFT was noted in the superior nasal region of the 5-6 mm zone of the scleral lens (mean [SD], 153.8 [96.4] $\mu \mathrm{m})$.

PLFT asymmetry was noted in different regions of the scleral lens, with a higher asymmetry in the $5-6 \mathrm{~mm}$ zone than the $2-5 \mathrm{~mm}$ zone. The greatest asymmetry in PLFT across the scleral lens opposing regions was observed in the inferotemporal versus superonasal region of the $5-6 \mathrm{~mm}$ zone with a mean difference of $168.8 \mu \mathrm{m}(\mathrm{P}<0.001)$. The variation in mean PLFT in different regions of the scleral lens is summarized in Table 1.

However, after 4 hours of scleral lens wear, the regional variation in PLFT across the different regions of the scleral lens was not statistically significant $(\mathrm{F}=4.692 ; \mathrm{P}=0.073)$. Although pairwise comparison revealed statistically significant differences in PLFT in the inferior, superior, superior nasal, and infeotemporal regions in the mid-peripheral (2-5 mm) zone compared with the central region $(\mathrm{P}<0.05)$. Likewise, there was a statistically significant difference noted in pairwise comparison in the superior, inferior, temporal, inferotemporal, and superior nasal regions in the peripheral zone (5-6 $\mathrm{mm})$ compared to the central $(\mathrm{P}<0.05)$.

The inferotemporal and the superonasal PLFT in the $5-6 \mathrm{~mm}$ zone of the scleral lens remained the thickest and the thinnest regions, respectively, after 4 hours of scleral lens wear.

\section{Scleral lens thickness}

The corrected thickness in the central 0-2 mm region of the scleral lens was $286.4 \pm 39.2 \mu \mathrm{m}$. The means of the corrected scleral lens thickness in different regions within the $6 \mathrm{~mm}$ of the scleral lens is presented in Table 1. The scleral lens thickness in the superior $2-5 \mathrm{~mm}$ region within the central $6 \mathrm{~mm}$ of the scleral lens was noted to be the thickest $(306.0 \pm 45.3 \mu \mathrm{m})$ followed by the superior 5-6 $\mathrm{mm}$ region with a thickness of $304.9 \pm 43.1 \mu \mathrm{m}$. The thinnest region within the central $6 \mathrm{~mm}$ of the scleral lens was noted in the superior-nasal region of the 5-6 mm zone of the scleral lens $(265.3 \pm$ $33.2 \mu \mathrm{m})$. However, this variation in the corrected scleral lens thickness within the central $6 \mathrm{~mm}$ was not statistically significant $(\mathrm{F}=3.366 ; \mathrm{P}=0.124)$.

\section{Corneal swelling after 4 hours of scleral lens wear}

Following 4 hours of scleral lens wear, a significant increase in corneal thickness was noted in all regions of the cornea compared with baseline measurements before scleral lens application ( $\mathrm{P}=$ 0.001). The greatest increase in corneal thickness was noted in the inferior region of the peripheral cornea $(5-6 \mathrm{~mm})$ zone with a mean increase of $14.2 \pm 10.8 \mu \mathrm{m}$, and the central corneal showed the least increase (mean increase, $6.3 \pm 7.3 \mu \mathrm{m}$ ).

Although the central 0-2 mm region of the cornea showed less corneal swelling compared with other peripheral regions of the cornea, the regional variation in corneal edema was not statistically significant $(\mathrm{F}=$ 3.346; $\mathrm{P}=0.126$ ). However, the pairwise comparison showed a significant difference in the induced corneal edema between the central versus the inferior regions in the mid-peripheral $(2-5 \mathrm{~mm})$ and peripheral zones $(5-6 \mathrm{~mm})$ of the cornea $(\mathrm{P}=0.048)$.

\section{Correlation between PLFT, regional corneal thickness, and corneal edema}

There was a fair relationship between the PLFT assessed after 4 hours of scleral lens application and

J Cont Lens Res Sci Vol 5(1):e32-e43; 17 December 2021

This article is distributed under the terms of the Creative Commons AttributionNon Commercial 4.0 International License. (C2021 Obinwanne CJ et al. 
TABLE 1 Mean \pm SD PLTT with Corresponding Corneal Edema Induced in Different Corneal Regions and the Correlation Coefficient of Corneal Edema versus Regional PLFT after 4 hours.

\begin{tabular}{|l|c|c|c|c|c|c|}
\hline Location & Initial-PLTT $(\boldsymbol{\mu m})$ & 4 hour-PLTT $(\boldsymbol{\mu m})$ & P-value & ${\text { Edema }(\boldsymbol{\%})^{\mathbf{a}}}$ & r value $^{\mathbf{b}}$ & SLT $(\boldsymbol{\mu m})$ \\
\hline 0-2 mm & $243.2 \pm 120.9$ & $205.5 \pm 134.6^{*}$ & 0.001 & $1.2 \pm 1.4$ & $0.468^{*}$ & $286.4 \pm 39.2$ \\
\hline I 2-5 mm & $295.6 \pm 134.7$ & $168.0 \pm 105.0^{*}$ & $<0.001$ & $2.0 \pm 1.6$ & $0.549^{*}$ & $273.0 \pm 40.1$ \\
\hline S 2-5 mm & $202.5 \pm 108.0$ & $270.5 \pm 167.2^{*}$ & 0.003 & $1.4 \pm 1.5$ & 0.081 & $306.0 \pm 45.3$ \\
\hline N 2-5 mm & $206.5 \pm 107.5$ & $172.3 \pm 95.8^{*}$ & $<0.001$ & $1.3 \pm 1.7$ & 0.272 & $278.0 \pm 33.9$ \\
\hline T 2-5 mm & $282.5 \pm 132.0$ & $241.1 \pm 132.3^{*}$ & $<0.001$ & $1.7 \pm 1.3$ & 0.411 & $293.0 \pm 38.6$ \\
\hline SN 2-5 mm & $193.5 \pm 102.3$ & $152.6 \pm 98.2^{*}$ & $<0.001$ & $1.2 \pm 1.7$ & 0.178 & $273.2 \pm 35.5$ \\
\hline ST 2-5 mm & $253.0 \pm 122.1$ & $195.6 \pm 114.8^{*}$ & $<0.001$ & $1.4 \pm 1.2$ & 0.374 & $291.9 \pm 47.2$ \\
\hline IN 2-5 mm & $251.1 \pm 111.9$ & $215.2 \pm 116.9^{*}$ & 0.011 & $1.8 \pm 1.8$ & 0.323 & $289.7 \pm 46.1$ \\
\hline IT 2-5 mm & $303.2 \pm 140.2$ & $266.6 \pm 142.6^{*}$ & 0.042 & $1.9 \pm 1.6$ & $0.447 *$ & $300.2 \pm 44.5$ \\
\hline I 5-6 mm & $309.1 \pm 139.7$ & $126.7 \pm 78.9^{*}$ & $<0.001$ & $2.6 \pm 1.9$ & 0.279 & $271.4 \pm 43.5$ \\
\hline S 5-6 mm & $174.1 \pm 104.0$ & $284.4 \pm 164.0^{*}$ & $<0.001$ & $2.0 \pm 1.9$ & -0.035 & $304.9 \pm 43.1$ \\
\hline N 5-6 mm & $176.3 \pm 97.2$ & $139.4 \pm 79.6^{*}$ & $<0.001$ & $1.3 \pm 2.0$ & 0.283 & $271.3 \pm 30.1$ \\
\hline T 5-6 mm & $304.3 \pm 137.1$ & $261.5 \pm 136.2^{*}$ & $<0.001$ & $1.9 \pm 1.4$ & 0.372 & $292.0 \pm 24.5$ \\
\hline SN 5-6 mm & $153.8 \pm 96.4$ & $104.4 \pm 66.0^{*}$ & $<0.001$ & $1.5 \pm 2.1$ & 0.314 & $265.3 \pm 33.2$ \\
\hline ST 5-6 mm & $241.6 \pm 122.1$ & $186.8 \pm 108.6^{*}$ & $<0.001$ & $1.6 \pm 1.6$ & 0.294 & $280.5 \pm 40.2$ \\
\hline IT 5-6 mm & $322.6 \pm 161.8$ & $286.7 \pm 149.4$ & 0.216 & $2.3 \pm 1.6$ & 0.153 & $300.7 \pm 41.4$ \\
\hline IN 5-6 mm & $253.6 \pm 115.9$ & $215.6 \pm 114.7^{*}$ & 0.017 & $2.0 \pm 2.4$ & 0.108 & $292.0 \pm 43.7$ \\
\hline
\end{tabular}

CT, corneal thickness; S, superior; I, inferior; $N$, nasal; T, temporal; IN, inferior nasal; IT, inferotemporal; SN, superonasal;

ST, superotemporal; SLT, scleral lens thickness; PLTT, post lens tear reservoir thickness.

${ }^{a}$ The percentage of corneal swelling after 4 hours of lens wear.

${ }^{b}$ PLTT at 4 hours versus corneal edema correlation coefficient.

*Statistically significant $(P<0.05)$.

the induced corneal edema in the $0-2 \mathrm{~mm}$ central cornea; this correlation was statistically significant $(\mathrm{r}=0.468 ; \mathrm{P}=0.037)$. In the $2-5 \mathrm{~mm}$ corneal region, the correlation between in PLFT assessed at 4 hours, and the induced corneal edema was not statistically significant in the regions within this zone, except for moderate correlation in the inferior $(\mathrm{r}=0.549$; $\mathrm{P}=0.012$ ) and fair correlation in the inferotemporal regions $(\mathrm{r}=0.447 ; \mathrm{P}=0.048)$. In the $5-6 \mathrm{~mm}$ region, the relationship between regional PLFT assessed at 4 hours and corneal edema was not statistically significant in any of the regions evaluated.

There was no correlation between corneal thickness in different regions of the cornea before scleral lens application and regional corneal edema in all the regions evaluated $(\mathrm{P}>0.05)$.

\section{Variation in scleral lens system $\mathrm{Dk} / \mathrm{t}$}

There was a statistically significant variation in the oxygen transmissibility of the scleral lens-tear reservoir system $(\mathrm{Dk} / \mathrm{t})$ across different regions within the central $6 \mathrm{~mm}$ of the scleral lens $(\mathrm{F}=48.472 ; \mathrm{P}=0.001)$. The mean $\mathrm{Dk} / \mathrm{t}$ of the system in the central 0-2 mm was $20.5 \pm 6.8 \mathrm{Fatt} \mathrm{Dk} / \mathrm{t}$ units. The super-nasal and infer-temporal quadrants in the 5-6 mm zone had the highest and least oxygen transmissibility in the regions studied, $26.8 \pm$ 7.1 and $16.4 \pm 4.6$ Fatt Dk/t units, respectively. The variation in the mean $\mathrm{Dk} / \mathrm{t}$ of the scleral lens tear reservoir system is summarized in Table 2. There was a negative moderate correlation between $\mathrm{Dk} / \mathrm{t}$ and corneal edema in the central region $(r=-0.528$; $\mathrm{P}<0.05)$, the inferior and temporal regions of the 
mid-peripheral zone $(\mathrm{r}=-0.531 ; \mathrm{P}<0.05$ and $\mathrm{r}=$ $-0.467 ; \mathrm{P}<0.05$, respectively), and the temporal region of the peripheral zone $(\mathrm{r}=-0.459 ; \mathrm{P}<0.05)$. The other correlations were fair to poor without statistical significance. The correlation between the scleral lens system $\mathrm{Dk} / \mathrm{t}$ and the induced corneal edema in different regions examined is summarized in Table 2.

\section{DISCUSSION}

In the present study, the thickest and thinnest PLFT were noted in the inferotemporal and superonasal regions within the $5-6 \mathrm{~mm}$ zone of the scleral lens, respectively. This finding differs from the study outcomes of other studies, where the thickest and thinnest PLFT were in the temporal and the nasal regions of the scleral lens, respectively. ${ }^{17,18}$ The difference in results maybe because of the morphological changes after scleral lens settling, variation in scleral topography, lens decentration, and difference in lens designs studied. Morphological changes in the conjunctiva and episcleral tissues after scleral lens wear has been shown in the superior region because of blinking and scleral lens compression. ${ }^{26}$ Scleral topography data indicates that the nasal region is notably the most elevated portion of the sclera, ${ }^{27}$ leading to temporal lens decentration. Studies investigating scleral lens decentration report an inferotemporal decentration which explains our findings. ${ }^{28,29}$ This may also account for the thinnest PLFT presence in the superonasal region. There is no data regarding scleral topography in Africans or whether ethnic variation in scleral topography exists; however, such differences, if present, may affect scleral lens centration and fit characteristics in different races.

However, after 4 hours of scleral lens wear, the variation in PLFT was no longer significant in most of the studied regions, which agreed with the previously reported Vincent et al.'s study ${ }^{18}$ Here, an initial regional variation in PLFT was noted immediately after lens application and plateaued in 90-120 minutes post lens application. Their study
TABLE 2 Mean Variation in the Scleral Lenstear Reservoir Thickness Oxygen Transmissibility $\left(\mathrm{Dk} / \mathrm{t}_{\mathrm{sLS}}\right)$ in the Different Regions and the Correlation between Dk/ $\mathrm{t}_{\mathrm{sLS}}$ and Corneal Edema.

\begin{tabular}{|l|c|c|}
\hline Location & Dk/t & r value $^{\mathbf{a}}$ \\
\hline 0-2 mm & $20.5 \pm 6.8$ & $-0.528^{*}$ \\
\hline I 2-5 mm & $22.5 \pm 6.6$ & $-0.531^{*}$ \\
\hline S 2-5 mm & $17.3 \pm 5.7$ & -0.060 \\
\hline N 2-5 mm & $22.0 \pm 6.7$ & -0.280 \\
\hline T 2-5 mm & $18.3 \pm 5.4$ & $-0.467^{*}$ \\
\hline SN 2-5 mm & $23.5 \pm 7.8$ & -0.189 \\
\hline ST 2-5 mm & $20.3 \pm 6.0$ & -0.417 \\
\hline IN 2-5 mm & $19.5 \pm 6.0$ & -0.386 \\
\hline IT 2-5 mm & $17.4 \pm 5.7$ & -0.423 \\
\hline I 5-6 mm & $24.8 \pm 6.3$ & -0.313 \\
\hline S 5-6 mm & $16.7 \pm 5.1$ & 0.097 \\
\hline N 5-6 mm & $24.0 \pm 6.5$ & -0.309 \\
\hline T 5-6 mm & $17.5 \pm 4.9$ & $-0.459^{*}$ \\
\hline SN 5-6 mm & $26.8 \pm 7.1$ & -0.226 \\
\hline ST 5-6 mm & $21.1 \pm 6.1$ & -0.332 \\
\hline IT 5-6 mm & $16.4 \pm 4.6$ & -0.189 \\
\hline IN 5-6 mm & $19.3 \pm 5.8$ & -0.193 \\
\hline
\end{tabular}

SLT, scleral lens thickness; $D \mathrm{k} / \mathrm{t}_{\text {SLS }}$ oxygen transmissibility of scleral lens tear reservoir system.

${ }^{a} \mathrm{Dk} / \mathrm{t}_{\text {SLS }}$ versus corneal edema correlation coefficient.

*Statistically significant $(P<0.05)$.

also noted that the superior nasal region of the most peripheral portion of the regional PLFT settled first at 90 minutes before other areas stabilized at 4 hours. Depicting those areas with thinner PLFT settled earlier than with thick PLFT. Interestingly, the thinnest PLFT in the present study was also the superior nasal region of the peripheral zone.

While some studies have reported less corneal edema after scleral lens wear than that seen in the present study, ${ }^{15,30}$ another study reported higher corneal edema (ranging from 1.5 to $4 \%$ ). ${ }^{16}$ The reason for such conflicting results maybe because of the oxygen transmissibility of the scleral lens used, tear reservoir thickness, duration of lens wear, and other possible reasons. 
Despite studies recommending lesser PLFT, ${ }^{24}$ evidence of scleral lenses with higher PLFT with minimal corneal edema abound. ${ }^{31-33}$ Such differences between experimental and clinical observation maybe because of the endothelial cell pump mechanism, ${ }^{34}$ tear exchange underneath the scleral lens, and seal-off after scleral lens settling. ${ }^{35}$ Even though a significant PLFT variation 10 minutes after scleral lens application and clinically significant variation was noted in some regions after 4 hours of wear, the variation in corneal edema was insignificant after 4 hours of lens wear, similar to the findings of another study. ${ }^{14}$ This finding maybe because regional variation in PLFT after scleral lens application does not last long enough to induce a measurable regional difference in corneal edema. This phenomenon may not hold for post graft corneas, as Kumar et al., noted statistically significant regional variations in edema induced after scleral lens wear in postpenetrating keratoplasty patients. More edema was noted close to the inferior graft-host junction. ${ }^{9}$

A fair to moderate correlation between the PLFT assessed 4 hours after lens application, corneal edema in the $0-2 \mathrm{~mm}$ (central) zone, and the inferior and inferior temporal regions in the 2-5 $\mathrm{mm}$ zone was noted in the present study. However, the relationship between regional PLFT and corneal edema after 4 hours of application was not statistically significant in the $5-6 \mathrm{~mm}$ peripheral corneal regions. This difference in the significance of corneal edema about the PLFT noted in different regions maybe because of a difference in endothelial cell count distribution in the cornea. Studies report increased endothelial cell count in the cornea periphery compared to the center in humans, ${ }^{34,36}$ this may leave the central cornea prone to corneal swelling in a hypoxic environment. Central endothelial cell count has been shown to positively correlate with central and peripheral corneal thickness in children ${ }^{37}$ and the elderly; ${ }^{36}$ hence corneal thickness may serve as an indicator for endothelial cell count health. However, the corneal thickness before scleral lens application did not correlate with induced corneal edema in the present study.
The classic oxygen transmissibility threshold to avoid hypoxia-induced central corneal edema during daily contact lens wear is 24.1 Fatt Dk/t units, as proposed by Holden and Mertz, ${ }^{38}$ which was later revised to 35 Fatt Dk/t by Harvitt and Bonanno. ${ }^{39}$ In the regions evaluated in the present study, only the inferior and the superornasal region of the 5-6 $\mathrm{mm}$ corneal zone attained the Holden and Mertz criterion, and none attained the Harvitt and Bonanno criteria. Not surprisingly, the superonasal portion of the 5-6 $\mathrm{mm}$ region had the thinnest PLFT and scleral lens thickness. Despite the low oxygen transmissibility calculated in this study's population. The highest mean percentage of corneal edema of $2.6 \%$ was noted in the inferior region of the $5-6 \mathrm{~mm}$ zone, which surprisingly had the second-highest scleral lens system oxygen transmissibility. This amount of corneal edema is less than the physiological cor-neal edema induced by sleep and still does not reach the level where it may be detected as corneal striae during routine slit lamp evaluation. ${ }^{40}$ To the best of our knowledge, this is the first article to report significant regional variation in oxygen transmissibility following scleral lens wear in a healthy population.

Although the variation in scleral lens thickness observed in this study was not statistically significant, other studies have reported quite the opposite by using the LaPlace method. ${ }^{41}$ Most likely because the area examined in this study was limited to the central $6 \mathrm{~mm}$ when the back optic zone diameter of the lens design used in this study was $8 \mathrm{~mm}$, hence minimal thickness variation may not be a surprising finding. In clinical practice, scleral lenses are fitted mostly on compromised corneas; post penetrating and lamellar keratoplasty, a steady decline in endothelial cell count greater than that seen in healthy eyes occurs $(7.8 \%$ vs. $0.52 \%){ }^{42,43}$ Hence, caution is required when fitting such eyes to avoid complications of corneal edema, and practitioners need to evaluate the PLFT in all regions and pay attention to the areas with greater PLFT.

This study does have some limitations; the large SD noted in the corneal edema may be because of the large SD in PLFT and scleral lens 
thickness measured. Diurnal variation in corneal thickness was not accounted for in this study; however, the magnitude of edema found was similar to that found in other studies that accounted for this bias. Ethnic differences in anterior scleral topography may exist and may also account for some differences noted in this study compared with others; we did not study such differences and hence requires concern in future studies. Scleral topography and lens decentration not measured in this study may have affected the results reported, as variation in scleral topography affects lens centration, leading to variation in PLFT. The lens decentration reflected in the study must have also affected the on-eye scleral lens thickness measurement; which could be the reason for the thinnest location of the lens noted in the superior-nasal 5-6 $\mathrm{mm}$ region instead of the center as expected. However, the thickness variation within the $6 \mathrm{~mm}$ region of the scleral lens was not statistically significant.

Thickness measurements underneath the scleral lens may be inaccurate because of the refractive index and power of a rigid scleral lens. ${ }^{44}$ Hence the correction equations were used in this study to adjust for the optical distortions caused by the above factors, which applies to the PLFT, and scleral lens thickness measured in this study.

Another limitation to be considered is that this study focused on the physiological changes in the cornea of healthy neophytes to scleral lens wear. Scleral lenses are mostly prescribed in compromised corneas, such as those with ectasia or post graft corneas. The study results may have varied if the study population consisted of participants with compromised corneas or with prior history of contact lens wear.

\section{CONCLUSION}

This study found significant regional differences in PLFT 10 minutes after scleral lens application but not after 4 hours of scleral lens wear. Despite a significant regional variation in oxygen transmissibility through the scleral lens, the induced corneal edema did not vary significantly in the studied regions.

\section{DECLARATION OF INTEREST}

The authors have no conflict of interests to disclose.

\section{FUNDING}

No external funding was received for this research.

\section{REFERENCES}

1. van der Worp E. A guide to scleral lens fitting. Version 2.0 [Monograph online]. Forest Grove, OR: Pacific University; 2015. Available from: http:// commons.pacificu.edu/mono/10/

2. Pearson RM, Efron N. Hundredth anniversary of August Muller's inaugural dissertation on contact lenses. Surv Ophthalmol. 1989;34:133-41. https:// doi.org/10.1016/0039-6257(89)90041-6

3. Ezekiel D. Gas permeable haptic lenses. J Br Contact Lens Assoc. 1983;6:157-61. https://doi. org/10.1016/S0141-7037(83)80064-0

4. Nixon AD, Barr JT, VanNasdale DA. Corneal epithelial bullae after short-term wear of small diameter scleral lenses. Cont Lens Anterior Eye. 2017;40:11626. https://doi.org/10.1016/j.clae.2016.11.007

5. Pullum KW, Stapleton FJ. Scleral lens induced corneal swelling: What is the effect of varying $\mathrm{Dk}$ and lens thickness? CLAO J. 1997;23:259-63. Available from: https://pubmed.ncbi.nlm.nih.gov/9348450/

6. Leung BK, Bonanno JA, Radke CJ. Oxygendeficient metabolism and corneal edema. Prog Retin Eye Res. 2011Nov;30(6):471-92. http://dx. doi.org/10.1016/j.preteyeres.2011.07.001

7. Maurice DM. The location of the fluid pump in the cornea. J Physiol. 1972;221(1):43-54. http://dx.doi. org/10.1113/jphysiol.1972.sp009736

8. Giasson CJ, Rancourt J, Robillard J, Melillo M, Michaud L. Corneal endothelial blebs induced in scleral lens wearers. Optom Vis Sci. 2019;96:810-17. https://doi.org/10.1097/OPX.0000000000001438

9. Kumar M, Shetty R, Khamar P, Vincent S. Scleral lens-induced corneal edema after penetrating 
keratoplasty. Optom Vis Sci. 2020;97:697-702. http://dx.doi.org/10.1097/OPX.0000000000001571

10. Murillo SE, Shariff A, Lass JH, Szczotka-Flynn LB. Acute corneal edema decades after penetrating keratoplasty for keratoconus in eyes wearing scleral contact lenses. Cont Lens Anterior Eye. 2021;44(1):108-14. https://doi.org/10.1016/j.clae.2020.10.008

11. Vincent SJ, Alonso-Caneiro D, Collins MJ. The time course and nature of corneal oedema during sealed miniscleral contact lens wear. Cont Lens Anterior Eye. 2019;42:49-54. https://doi. org/10.1016/j.clae.2018.03.001

12. Efron N. Intersubject variability in corneal swelling response to anoxia. Acta Ophthalmol (Copenh). 1986;64:302-5. https://doi. org/10.1111/j.1755-3768.1986.tb06924.x

13. Kim YH, Tan B, Lin MC, Radke CJ. Central corneal edema with scleral-lens wear. Curr Eye Res. 2018;43(11):1305-15. http://dx.doi.org/10.1080/027 13683.2018.1500610

14. Vincent SJ, Alonso-Caneiro D, Collins MJ, Beanland A, Lam L, Lim CC, et al. Hypoxic corneal changes following eight hours of scleral contact lens wear. Optom Vis Sci. 2016 Mar;93:293-9. https://doi.org/10.1097/OPX.0000000000000803

15. Vincent SJ, Alonso-Caneiro D, Collins MJ. Corneal changes following short-term miniscleral contact lens wear. Cont Lens Anterior Eye. 2014;37:461-8. https://doi.org/10.1016/j.clae.2014.08.002

16. Compañ V, Oliveira C, Aguilella-Arzo M, Mollá S, Peixoto-de-Matos SC, González-Méijome JM. Oxygen diffusion and edema with modern scleral rigid gas permeable contact lenses. Invest Ophthalmol Vis Sci. 2014;55:6421-9. https:/doi. org/10.1167/iovs.14-14038

17. Nau CB, Schornack MM. Region-specific changes in post lens fluid reservoir depth beneath small-diameter scleral lenses over 2 hours. Eye Contact Lens. 2018;44:210-15. https://doi.org/10.1097/ ICL.0000000000000382

18. Vincent SJ, Alonso-Caneiro D, Collins MJ. Regional variations in postlens tear layer thickness during scleral lens wear. Eye Contact Lens. 2019;10:1-7. https://doi.org/10.1016/j.clae.2019.10.025

19. Alonso-Caneiro D, Read SA, Vincent SJ, Collins MJ, Wojtkowski M. Tissue thickness calculation in ocular optical coherence tomography.
Biomed Opt Express. 2016;7(2):629-45. https://doi. org/10.1364/BOE.7.000629

20. Hosseini H, Nilforushan N, Moghimi S, Bitrian E, Riddle J, Lee GY, et al. Peripapillary and macular choroidal thickness in glaucoma. J Ophthalmic Vis Res. 2014;9(2):154-61. Available from: https:// pubmed.ncbi.nlm.nih.gov/25279115/

21. Lee EC, de Boer JF, Mujat M, Lim H, Yun SH. In vivo optical frequency domain imaging of human retina and choroid. Opt Express. 2006;14(10):440311. https://doi.org/10.1364/OE.14.004403

22. Ramasubramanian V, Glasser A. Distortion correction of visante optical coherence tomography cornea images. Optom Vis Sci. 2015;92(12):1170-81. http://dx.doi.org/10.1097/OPX.0000000000000725

23. Weissman BA, Ye P. Calculated tear oxygen tension under contact lenses offering resistance in series: Piggyback and scleral lenses. Cont Lens Anterior Eye. 2006;29(5):231-7. http://dx.doi. org/10.1016/j.clae.2006.09.001

24. Michaud L, van der Worp E, Brazeau D, Warde $\mathrm{R}$, Giasson CJ. Predicting estimates of oxygen transmissibility for scleral lenses. Cont Lens Anterior Eye. 2012 Dec;35(6):266-71. http://dx.doi. org/10.1016/j.clae.2012.07.004

25. Benjamin WJ. Oxygen transport through contact lenses. In: Guillon M, Ruben M, editors. Contact lens practice. London: Chapman Hall Medical Publishers; 1994. p. 47-69.

26. Consejo A, Llorens-Quintana C, Bartuzel MM, Iskander DR, Rozema JJ. Rotation asymmetry of the human sclera. Acta Ophthalmol. 2019;97:26670. https://doi.org/10.1111/aos.13901

27. Vincent SJ, Alonso-Caneiro D, Collins MJ. The temporal dynamics of miniscleral contact lenses: Central corneal clearance and centration. Cont Lens Anterior Eye. 2018;41:162-8. https://doi. org/10.1016/j.clae.2017.07.002

28. Visser ES, Van der Linden BJ, Otten HM, Van der Lelij A, Visser R. Medical applications and outcomes of bitangential scleral lenses. Optom Vis Sci. 2013;90:1078-85. https://doi.org/10.1097/ OPX.0000000000000018

29. Otchere H, Jones LW, Sorbara L. Effect of time on scleral lens settling and change in corneal clearance. Optom Vis Sci. 2017;94:908-13. https://doi. org/10.1097/OPX.0000000000001111 
30. Frisani M, Beltramo I, Greco M. Changes in corneal thickness by miniscleral contact lenses. Cont Lens Anterior Eye. 2015;38:38-9. https://doi. org/10.1016/j.clae.2014.11.064

31. Sonsino J, Mathe DS. Central vault in dry eye patients successfully wearing scleral lens. Optom Vis Sci. 2013;90:e248-51. https://doi.org/10.1097/ OPX.0000000000000013

32. Fisher D, Collins MJ, Vincent SJ. Fluid reservoir thickness and corneal edema during open-eye scleral lens wear. Optom Vis Sci. 2020;97(9):683-9. http://dx.doi.org/10.1097/OPX.0000000000001558

33. Rathi VM, Mandathara PS, Dumpati S, Sangwan VS. Change in vault during scleral lens trials assessed with anterior segment optical coherence tomography. Cont Lens Anterior Eye. 2017;40:157-61. https://doi.org/10.1016/j. clae.2017.03.008

34. Sturrock GD, Sherrard ES, Rice NSC. Specular microscopy of the corneal endothelium. $\mathrm{Br} \mathrm{J}$ Ophthalmol. 1978;62:809-14. https://doi.org/10.1136/ bjo.62.12.809

35. Alonso-Caneiro D, Vincent SJ, Collins MJ. Morphological changes in the conjunctiva, episclera and sclera following short-term miniscleral contact lens wear in rigid lens neophytes. Cont Lens Anterior Eye. 2016;39:53-61. https://doi.org/10.1016/j. clae.2015.06.008

36. Müller A, Craig JP, Grupcheva CN, McGhee CNJ. The effects of corneal parameters on the assessment of endothelial cell density in the elderly eye. Br J Ophthalmol. 2004;88:325-30. https://doi. org/10.1136/bjo.2003.019315

37. Müller A. Assessment of eye growth-related changes in the corneal endothelium of children and young teenagers. PhD thesis, Glasgow Caledonian University, 2000.

38. Holden BA, Mertz GW, McNally JJ. Corneal swelling response to contact lenses worn under extended wear conditions. Investig Ophthalmol Vis Sci. 1983;24:218-26. Available from: https://iovs.arvojournals.org/article.aspx?articleid=2159618

39. Harvitt DM, Bonanno JA. Re-evaluation of the oxygen diffusion model for predicting minimum contact lens $\mathrm{Dk} / \mathrm{t}$ values needed to avoid corneal anoxia. Optom Vis Sci. 1999;76(10):712-19. http:// dx.doi.org/10.1097/00006324-199910000-00023

40. Polse KA, Mandell RB. Etiology of corneal striae accompanying hydrogel lens wear. Invest Ophthalmol Vis Sci. 1976;15:553-6. Available from: https://pubmed.ncbi.nlm.nih.gov/931704/

41. Vincent SJ, Alonso-Caneiro D, Kricancic H, Collins MJ. Scleral contact lens thickness profiles: The relationship between average and centre lens thickness. Cont Lens Anterior Eye. 2019;42(1):5562. http://dx.doi.org/10.1016/j.clae.2018.03.002

42. BourneWM.Cellularchangesintransplantedhuman corneas. Cornea. 2001Aug;20(6):560-9. http://dx. doi.org/10.1097/00003226-200108000-00002

43. Bourne WM, Hodge DO, Nelson LR. Corneal endothelium five years after transplantation. Am J Ophthalmol. 1994 Aug;118(2):185-96. http://dx. doi.org/10.1016/s0002-9394(14)72898-3

44. Vincent SJ, Alonso-Caneiro D, Collins MJ. Optical coherence tomography and scleral contact lenses: Clinical and research applications. Clin Exp Optom. 2019;102:224-41. https://doi.org/10.1111/ cxo.12814 